\title{
LOCALIZAÇÃO DE INFORMAÇÕES NA INTERNET: CARACTERÍSTICAS E FORMAS DE FUNCIONAMENTO DOS MECANISMOS DE BUSCA
}

Regina Meyer BRANSKI

RESUMO

Este trabalho apresenta diferentes formas de operação dos diversos pesquisadores na Internet, demonstrando como funcionam os mecanismos de busca, tais como a Alta Vista, BookMark, Cadê, Infoseek, etc., fazendo uma comparação entre os diversos critérios e ferramentas de acesso, demonstrando as características dos catálogos e indices sites em Português e Inglês. Discute, de forma breve, princípios para a localização de informações na Internet.

Palavras-chave: Internet; mecanismo de busca.

\section{ABSTRACT}

This paper presents different operation ways of several researchers in the Internet, demonstrating how the search mechanisms work, such as Alta Vista, Bookmark, Cadê, Infossek etc., comparing the different access instruments, demonstrating the characteristics of catalogues and sites indexes in Portuguese and English. It briefly discusses principles to access information using the Internet.

Keywords: Internet; search engines.

\section{INTRODUÇÃO}

A Internet é um conjunto de inúmeras redes de computadores, conectadas entre si, que permite a comunicação, partilha de informações, programas e equipamentos entre seus usuários. Existem, atualmente na Internet, milhares de páginas cobrindo os mais variados assuntos e interesses. O conjunto das páginas de uma empresa ou instituição é chamado de site. Cada página tem um endereço exclusivo em formato conhecido chamado Uniform Resource Locator $(U R L)$ que possibilita sua localização por computadores de todo o mundo.
Localizar informações na Internet, entretanto, pode parecer uma tarefa impossível. Isto não só pelo grande volume de páginas disponíveis como, também, pelo seu caráter anárquico. Os documentos não estão organizados segundo um padrão determinado como, por exemplo, as bibliotecas e encontrar a informação desejada depende, principalmente, da utilização eficiente das ferramentas de busca disponíveis.

Este texto pretende mostrar as diferenças nas formas de operação dos diversos pesquisadores atualmente existentes na Internet e como podem afetar os resultados das buscas realizadas. 
Conhecendo suas características e modo de funcionamento é possível extrair todo o potencial de cada ferramenta e, deste modo, obter melhores resultados na localização das informações desejadas.

\section{O QUE SÄO MECANISMOS DE BUSCA E COMO FUNCIONAM}

Pesquisadores ou mecanismos de busca são sites especializados em localizar informações na Internet. Digita-se o termo procurado, geralmente uma palavra ou frase, numa caixa em branco disponibilizada no site e, em seguida, solicita-se que a busca seja efetuada. Os pesquisadores procuram a ocorrência deste termo em seus bancos de dados e apresentam os resultados na forma de uma lista de documentos da Internet que contém a palavra ou palavras pesquisadas.

Os pesquisadores podem ser enquadrados em duas amplas categorias: catálogos por assunto e índices. A principal diferença entre eles é a forma como seus bancos de dados são compilados.

Nos catálogos as informações são organizadas e classificadas em categorias temáticas com a ajuda de pessoas. O interessado em ter seu site catalogado envia uma breve descrição do conteúdo, solicitando a inclusão de seu endereço Internet no banco de dados. Os editores poderão ou não aceitar a inclusão. Em caso afirmativo, classificarão o endereço na categoria que julgarem mais adequada.

Os catálogos limitam-se a verificar a ocorrência do termo pesquisado na descrição enviada pelo autor, não considerando o texto integral do site. Um dos catálogos mais populares da Internet é o Yahoo (http://www.yahoo.com ).

Os índices, por sua vez, criam seus bancos de dados automaticamente, indexando as informações sem qualquer classificação. Seus bancos de dados são compostos não só através de solicitações enviadas pelos autores, como também, captando as informações através de programas conhecidos como spiders ou aranhas.

Estes programas vasculham a Internet visitando os sites, lendo seu conteúdo e seguindo seus links para outras páginas. Alguns índices indexam integralmente o conteúdo dos sites, outros somente o título e um resumo algoritmicamente construído, outros o título e as primeiras linhas do site. De toda forma, cada endereço encontrado é registrado e passa a fazer parte do banco de dados da ferramenta de pesquisa.

Os spiders iniciam sua busca, geralmente, a partir de uma relação de páginas mais populares ou consideradas melhores. Seguem os links destas páginas para encontrar mais links e ir, sucessivamente, adicionando os endereços ao banco de dados. Volta aos sites em intervalos regulares, a cada um ou dois meses, para verificar alterações e manter o sistema atualizado. Um dos índices mais utilizados na rede é o Alta Vista (http://www.altavista.digital.com).

Embora os índices indexem tudo que forem capazes de encontrar, prevalecem páginas de origem americana. Além disso, suas interfaces são, no geral, em inglês e seus serviços focados na audiência dos EUA. Por isto alguns índices disponibilizam edições regionais.

Ediçãoregional é um serviço especial criado por algumas ferramentas de busca para servir determinadas regiões ou países. Alguns pesquisadores utilizam-se de seu banco de dados original, apresentando somente o resultado no idioma utilizado no país (mirror sites). Outros filtram os sites pelo domínio (país de origem) construindo novos bancos de dados restritos a países específicos (filter). Outros, ainda, detectam o país de origem do visitante e apresentam uma página com informações específicas para aquele usuário. Somente os índices Alta Vista (mirror) e Infoseek (filter) (http://www.infoseek.com) oferecem edições regionais para o Brasil.

Todos os mecanismos de busca apresentam o resultado da pesquisa na forma de links de hipertextos. Isto é, clicando-se com o mouse sobre um dos documentos listados, o próprio documento, que está fora do banco de dados do pesquisador, é trazido para o computador do usuário.

Diferentemente dos humanos, os mecanismos de busca são incapazes de formular perguntas adicionais que definam melhor o objeto ou de se valer de suas experiências anteriores para escolher, entre os documentos encontrados, os mais relevantes. Assim, para uma maior eficiência, utilizam critérios que envolvem localização e freqüência da expressão procurada. 
Verificam a existência dos termos buscados no título, nas primeiras linhas e o número de ocorrências. A partir deste levantamento definem a localização de cada documento na relação apresentada como resposta.

Embora os mecanismos de busca coletem as informações basicamente do mesmo modo, os resultados apresentados numa consulta podem diferir grandemente de uma ferramenta para outra. Esta diferença é decorrente dos critérios utilizados para construção do banco de dados e das formas de funcionamento de cada ferramenta:

- a relação de páginas iniciais a partir do qual o spider percorrerá a rede em busca de informação;

- as informações enviadas pelos autores que escolhem as ferramentas onde pedirão a inclusão;

- de como indexam as informações de cada site (se armazena o texto integral, se somente o título e um pequeno resumo algoritmicamente construído do conteúdo, se o título e as primeiras linhas do site, etc.) e

- no caso dos catálogos, os critérios humanos utilizados para a indexação e classificação das informações.

Assim, a mesma pesquisa em diferentes mecanismos de busca pode produzir resultados bastante diversos. Deve-se considerar, ainda, que os pesquisadores não incluem em seus bancos de dados todos os sites existentes na Internet. Operam a partir de suas próprias bases compostas de sites, textos e descrições selecionados a partir da totalidade dos documentos da rede. A utilização de mais de um pesquisador garante, portanto, uma maior cobertura e, possivelmente, um resultado mais satisfatório.

\section{REFINANDO A PESQUISA}

A maioria dos pesquisadores oferece a possibilidade de refinamento da pesquisa. Através de certos comandos, que podem variar de ferramenta para ferramenta, é possível definir melhor o objeto de interesse e tornar a pesquisa mais eficiente.

Algumas ferramentas trabalham com um sistema chamado lógica booleana que utiliza conceitos matemáticos. Empregando-se a expressão AND entre os termos pesquisados a ferramenta retornará somente os endereços onde estão presentes todos os termos da pesquisa. Com OR os sites selecionados conterão pelo menos uma das palavras solicitadas. Outras alcançam resultados similares utilizando os sinais +/- entre os termos. É possível, ainda, a localização de frases exatas (entre aspas), de arquivos de imagem, de páginas com títulos específicos, a utilização de linguagem natural (digitar uma pergunta e solicitar a resposta), a limitação da busca pelo domínio etc.

O conhecimento dos refinamentos aceitos pelos diferentes mecanismos de busca melhora a eficiência de uma pesquisa. É possível, determinando os termos para a busca, avaliar quais os pesquisadores mais adequados e que, portanto, podem contribuir de fato para o sucesso da pesquisa.

PESQUISANDO NA INTERNET: ESTRATÉGIAS, ANÁLISE DO ASSUNTO E ESCOLHA DA FERRAMENTA DE PESQUISA

Quando se utiliza uma ferramenta de busca na Internet, a pesquisa limita-se ao banco de dados daquela ferramenta. O sucesso da pesquisa depende da habilidade em encontrar o melhor mecanismo de busca para o objetivo pretendido e a capacidade de extrair todo seu potencial utilizando formas de refinamento.

Uma análise prévia do assunto permite determinar quais os termos mais adequados e as melhores ferramentas. Na tabela 1, a seguir, estão descritas as principais características de catálogos e índices com sites em Português. Na tabela 2, em Inglês. A tabela 3, elaborada pelo UC Berkeley Library, estabelece de modo competente a relação entre necessidades e características dos pesquisadores. À direita estão relacionados os possíveis objetivos da pesquisa e, à esquerda, os refinamentos adequados àquela necessidade.

\section{ESTRATÉGIAS NĀO RECOMENDADAS}

Pela pouca eficiência na obtenção de resultados não são recomendáveis as seguintes abordagens para localizar documentos na Web: 
Tabela 1. Características dos catálogos e índices sites em Português

\begin{tabular}{|c|c|c|c|c|c|c|c|}
\hline & Alta Vista & Bookmark & Cadê? & Infoseek & Onde ir? & Radar UOL & Surf \\
\hline Categoria & Índice & Índice & Catálogo & Índice & Catálogo & Índice & Índice e \\
\hline Abrangência & $\begin{array}{l}110 \text { milhões de } \\
\text { páginas }\end{array}$ & 600.000 páginas & 45.000 sites & $\begin{array}{c}60 \text { milhões de } \\
\text { páginas }\end{array}$ & NI & $\begin{array}{l}54 \text { milhões de } \\
\text { páginas }\end{array}$ & $\begin{array}{c}\text { Catálogo } \\
50.000 \text { sites }\end{array}$ \\
\hline Indexa texto completo? & Sim & Sim & Não & Sim & Não & Sim & Sim \\
\hline Busca em línguas específicas? & Sim & Não & Não & Sim & Não & $\mathrm{Sim}^{2}$ & Não \\
\hline Procura frase exata? & Sim & Sim & Sim & Sim & Não & Sim & Não \\
\hline Aceita linguagem natural?! & Sim & Não & Não & Sim & Não & Não & Não \\
\hline Faz pesquisa avançada (boolena)? & Sim & Sim & Não & Sim & Não & Sim & Sim \\
\hline Procura por nome de domínio? & Sim & Não & Não & $\operatorname{Sim}$ & Não & Sim & Não \\
\hline Procura imagens? & Sim & Não & Não & Sim & Não & Sim & Não \\
\hline Procura links? & Sim & Não & Não & Sim & Não & Sim & Não \\
\hline Procura por data? & Sim & Não & Não & Não & Não & Sim & Não \\
\hline Diferencia maiúscula e minúscula & Sim & Não & Não & Sim & Não & $\mathrm{Sim}^{3}$ & Não \\
\hline Número de sites regionais & 4 & - & - & 10 & - & - & - \\
\hline É possível pedir inclusão de site? & Sim & Não & Sim & Sim & Sim & Sim & Sim \\
\hline
\end{tabular}

${ }^{1}$ Por exemplo: Quando Santos Dumont inventou o avião?

${ }^{2}$ Somente Português

${ }^{3}$ Apenas em alguns casos

NI: não informa

Fonte: Revista Info Exame - janeiro de 1998

Tabela 2. Características dos catálogos e índices sites em Inglês

\begin{tabular}{|c|c|c|c|c|c|c|c|c|}
\hline & Alta Vista & Excite & HotBot & Infoseek & Lycos & \begin{tabular}{|c|}
$\begin{array}{c}\text { Northern } \\
\text { Light }\end{array}$ \\
\end{tabular} & Web Crawler & Yahoo! \\
\hline Categoria & Índice & Índice e Catálogo & Índice & $\begin{array}{l}\text { Índice e } \\
\text { Catálogo }\end{array}$ & $\begin{array}{c}\text { Índicee } \\
\text { Catálogo }\end{array}$ & Índice & $\begin{array}{l}\text { Índice e } \\
\text { Catálogo }\end{array}$ & $\begin{array}{l}\text { Índicee } \\
\text { Catálogo }\end{array}$ \\
\hline Abrangência & $\begin{array}{c}110 \text { milhões } \\
\text { de páginas }\end{array}$ & $\begin{array}{c}50 \text { milhões de } \\
\text { páginas }\end{array}$ & $\begin{array}{l}54 \text { milhões } \\
\text { de páginas }\end{array}$ & $\begin{array}{l}60 \text { milhões } \\
\text { de páginas }\end{array}$ & $\begin{array}{l}30 \text { milhões de } \\
\text { páginas }\end{array}$ & $\begin{array}{l}50 \text { milhões } \\
\text { de páginas }\end{array}$ & 5000 sites & NI \\
\hline Indexa texto completo? & Sim & Sim & Sim & Sim & Sim & Sim & Sim & Sim \\
\hline Busca em línguas específicas? & Sim & Sim & Não & Nāo & Não & Não & Não & Não \\
\hline Procura frase exata? & Sim & Sim & Sim & Sim & Sim & Sim & Sim & Sim \\
\hline Aceita linguagem natural? ${ }^{1}$ & Sim & Sim & Não & Sim & Sim & Não & Sim & Sim \\
\hline Faz pesquisa avançada (boolena)? & Sim & $\operatorname{Sim}$ & Sim & Sim & Sim & Sim & Sim & Sim \\
\hline Procura por nome de domínio? & Sim & Não & Sim & Sim & Não & Não & Não & Não \\
\hline Procura imagens? & Sim & Não & Sim & Sim & Sim & Não & Não & Não \\
\hline Procura links? & Sim & Não & Sim & Sim & Não & Não & Não & Não \\
\hline Procura por data? & Sim & Não & Sim & Não & Não & Não & Não & $\operatorname{Sim}$ \\
\hline Diferencia maiúscula e minúscula & Sim & Não & $\mathrm{Sim}^{3}$ & Sim & Não & Não & Não & Sim \\
\hline Número de sites regionais & 4 & 7 & - & 10 & 9 & - & & 23 \\
\hline É possível pedir inclusão de site? & Sim & Sim & Sim & Sim & Sim & Sim & Sim & Sim \\
\hline
\end{tabular}

1 Por exemplo: Quando Santos Dumont inventou o avião?

${ }^{2}$ Somente Português

${ }^{3}$ Apenas em alguns casos

NI: não informa

Fonte: Revista Info Exame - janeiro de 1998 
- Exploração de catálogos. Localiza-se documentos tentando combinar o assunto pesquisado com a categoria mais geral de uma hierarquia de assuntos. Vai-se, então, escolhendo subcategorias que, se supõem, levem ao objetivo pretendido. A principal dificuldade é determinar sob qual categoria o assunto está classificado. A categoria "saúde", por exemplo, pode conter documentos sobre medicina, homeopatia, psiquiatria e esporte em determinado catálogo. Em outro, "medicina" pode incluir saúde, saúde mental e medicina alternativa e pode classificar esporte sob a categoria "estilo de vida".

- Palavras-chave simples em bancos de dados amplos. Pesquisa simples com palavras- chave é buscar uma ou mais palavras, separadas por espaços, na caixa inicial em branco que se encontra em qualquer ferramenta de pesquisa. Esta forma de busca recupera todos os endereços do banco de dados que contenham a palavra ou palavras pesquisadas. Em banco de dados extensos gera excesso de documentos sendo, grande parte, sem relevância. Neste caso é aconselhável utilizar técnicas mais avançadas de pesquisa. Nos bancos de dados menores e em catálogos por assunto, entretanto, pesquisas utilizando palavras chaves simples podem fornecer uma boa aproximação.

\section{OUTRAS FERRAMENTAS DISPONIVEIS NA INTERNET PARA LOCALIZAR INFORMAÇŌES}

\section{Metapesquisadores}

Nas ferramentas de pesquisa mais comuns (tais como Infoseek, Alta Vista, Yahoo!, Hotbot, etc.), submetem-se as palavras a um único banco de dados e recebe-se uma relação dos documentos onde constem as palavras pesquisadas. Os resultados obtidos em diferentes pesquisadores podem variar bastante, mas também podem conter vários resultados duplicados.

Os metapesquisadores buscam, simultaneamente, em vários mecanismos de busca. Não possuem banco de dados próprio e funcionam como um agente intermediário que repassa a pesquisa, obtêm as respostas dos pesquisadores individualmente e, então, apresenta um resultado unificado, extraído das diversas fontes. Em poucos segundos os metapesquisadores compilam os resultados obtidos, economizando tempo e fornecendo uma visão geral do tipo de documentos armazenados em cada ferramenta.

A utilização dos metapesquisadores não elimina a necessidade de conhecer as características individuais dos diversos mecanismos de busca. Quanto mais se conhece sobre as formas de funcionamento das ferramentas que os alimentam, melhor julgamento quanto a confiabilidade dos resultados obtidos $\mathrm{Se}$, por exemplo, a pesquisa exige determinados refinamentos não processáveis pelas ferramentas que constituem o metapesquisador, podem resultar erros e resultados inadequados.

Todos os metapesquisadores listados no texto produzem bons resultados e tem certas características em comum:

- buscam nos pesquisadores mais populares;

- são rápidos por utilizarem pesquisa em paralelo (simultânea) e possuírem processadores de alta velocidade para formatar e apresentar o resultado;

- permitem determinação do tempo máximo de espera e personalização de alguns aspectos do formato.

Diferem em outras:

- na forma de compilação dos resultados. Alguns apresentam o resultado de cada pesquisador em seqüência, fornecendo uma lista na ordem em que foi feita a pesquisa. Outros analisam os resultados eliminando as duplicações. Em alguns casos pode-se especificar como o resultado será organizado e, em outros, é organizado automaticamente em frases ou palavras significativas.

- na capacidade de manipular pesquisas complexas. Quando um metapesquisador envia determinada forma de refinamento para um mecanismo de busca que não a processe, pode gerar erros e resultados confusos. 
- na customização da estratégia de pesquisa. Alguns possuem maior flexibilidade para alterar o limite de tempo de espera e escolher a apresentação do resultado. Outros permitem especificar quais ferramentas de pesquisa serão utilizadas e em que ordem.

No quadro abaixo (tabela 4) estão comparadas as características de alguns metapesquisadores:

Tabela 3. Necessidades X Características das Ferramentas de Pesquisa

\begin{tabular}{|c|c|}
\hline $\begin{array}{l}\text { Procurando um nome próprio ou frase exata? } \\
\text { - Nome de uma organização, sociedade ou movimento } \\
\text { - Nome próprio ou um indivíduo } \\
\text { - Sequiência de palavras precisas associadas, geralmente, a um assunto } \\
\text { É possível pensar em uma organização, nome próprio ou frase para pesquisar? } \\
\text { Passo inicial para localizar o que se procura. }\end{array}$ & $\begin{array}{l}\text { Pesquisador deve ser capaz de localizar frases. Exige que todos os termos } \\
\text { apareçam na ordem exata em que foram digitados. } \\
\text { Coloque a frase entre aspas. Exemplo: } \\
\text { - "world health organization" } \\
\text { - "regina meyer branski" } \\
\text { - "comércio internacional" }\end{array}$ \\
\hline $\begin{array}{l}\text { Os termos procurados são palavras comuns com muitos significados e vários } \\
\text { contextos? } \\
\text { - criança associada à televisão e à violência }\end{array}$ & $\begin{array}{l}\text { Utilizar AND pode ajudar } \\
\text { - criança and televisão and violência } \\
\text { - + criança + televisão + violência } \\
\text { Ou utilize outros termos que possam levar ao mesmo assunto: } \\
\text { - + jornalismo + ética + censura } \\
\text { Nova pesquisa ou controle dos termos resultantes podem também ser úteis } \\
\text { - Após a pesquisa, submeta o resultado a outros aspectos }\end{array}$ \\
\hline $\begin{array}{l}\text { O resultado apresenta inúmeros termos que você não quer? } \\
\text { - Pesquisa por engenharia biomédica e câncer traz inúmeros programas } \\
\text { acadêmicos e o que se procura são artigos. }\end{array}$ & $\begin{array}{l}\text { Utilizar AND NOT pode ajudar } \\
\text { - "engenharia biomédica" AND câncer AND NOT "departamento de" } \\
\text { AND NOT "escola de } \\
\text { Ou seu equivalente } \\
\text { - + "engenharia biomédica" + câncer = "departamento de" - "escola de" }\end{array}$ \\
\hline $\begin{array}{l}\text { Existem sinônimos, variações de ortografia ou palavras estrangeiras para o que } \\
\text { se está buscando? } \\
\text { - Women, females com networking } \\
\text { - Sarajevo, Sarayevo com peace } \\
\text { - Literature, litterature com French, francaise }\end{array}$ & $\begin{array}{l}\text { Utilizar OR } \\
\text { - (Women OR female) AND networking } \\
\text { - (Sarajevo OR Sarayevo) AND peace } \\
\text { - (literature OR litterature) AND (French OR francaise) } \\
\text { Ou seu equivalente. Neste caso, a ausência de parâmetros será entendida } \\
\text { como OR. } \\
\text { - + networking women females } \\
\text { - + peace Sarajevo Sarayevo } \\
\text { - literature litterature + French } \\
\text { - literature litterature + francaise }\end{array}$ \\
\hline $\begin{array}{l}\text { Está procurando por Home Pages e/ou outros documentos básicos sobre os } \\
\text { termos? } \\
\text { - Home Page da American Dietetic Association } \\
\text { - Páginas básicas sobre comércio exterior }\end{array}$ & $\begin{array}{l}\text { Limite a pesquisa ao título } \\
\text { - title: "American Dietetic Association" } \\
\text { - title: "comércioexterior" }\end{array}$ \\
\hline $\begin{array}{l}\text { Procurando termos com vários finais possíveis? } \\
\text { - feminismo, feminista, feminino } \\
\text { - crianças, criança, criançada }\end{array}$ & $\begin{array}{l}\text { É possível adaptar todas as variações em um único termo de pesquisa } \\
\text { - femini* } \\
\text { - criança* }\end{array}$ \\
\hline $\begin{array}{l}\text { Letras maiúsculas } \mathrm{X} \text { minúsculas } \\
\text { - letras minúsculas sempre recuperam as maiúsculas. As maiúsculas } \\
\text { identificam somentesuassemelhantes. }\end{array}$ & $\begin{array}{l}\text { Alguns pesquisadores diferenciam as letras minúsculas das maiúsculas. } \\
\text { Outros não. Utilizando-se letras maiúsculas a pesquisa pode recuperar } \\
\text { somente letras semelhantes. Se, entretanto, utilizam-se minúsculas sempre } \\
\text { recuperam as formas maiúsculas. }\end{array}$ \\
\hline
\end{tabular}

Extraído dosite da Universidade da Califórnia, Berkeley

Fonte:http://www.lib.berkeley.edu/TeachingLib/Guides/Internet/ThingsToKnow.html 
Tabela 4. Características de alguns metapesquisadores

\begin{tabular}{|c|c|c|c|c|}
\hline & Dogpile & Inference Find & MetaCrawler & Metafind \\
\hline $\begin{array}{l}\text { Ferramentas de } \\
\text { busca utilizadas }\end{array}$ & $\begin{array}{c}\text { Alta Vista, Excite, } \\
\text { Excite Subject Guide, } \\
\text { HotBot,Lycos } \\
\text { Infoseek, Lycos a2z, } \\
\text { Magellan, } \\
\text { PlanetSearch, WWW } \\
\text { Worm, Yellow Pages, } \\
\text { WebCrawler, What-U-Seek, } \\
\text { Yahoo! }\end{array}$ & $\begin{array}{c}\text { Alta Vista, Excite Search, } \\
\text { Infoseek, Lycos, } \\
\text { WebCrawler, Yahoo! }\end{array}$ & $\begin{array}{l}\text { Alta Vista, Excite, } \\
\text { Infoseek, Lycos, } \\
\text { WebCrawler, Yahoo! }\end{array}$ & $\begin{array}{c}\text { Alta Vista, Excite, } \\
\text { HotBot, Infoseek, Open } \\
\text { Text, WebCrawler. }\end{array}$ \\
\hline $\begin{array}{l}\text { Apresentação do } \\
\text { resultado }\end{array}$ & $\begin{array}{l}\text { Sequiência. Lista os links } \\
\text { após cada ferramenta } \\
\text { pesquisada. Não possui uma } \\
\text { classificação própria. A } \\
\text { classificação utilizada é a da } \\
\text { própria ferramenta de } \\
\text { pesquisa podendo ocorrer } \\
\text { duplicações. Se nas primeiras } \\
\text { três ferramentas pesquisadas } \\
\text { o resultado for superior a } 10 \\
\text { links, apresenta, como opção, } \\
\text { a possibilidade de buscar } \\
\text { outras ferramentas }\end{array}$ & $\begin{array}{c}\text { Classificados em } \\
\text { agrupamentos de palavras } \\
\text { ou frases encontradas no } \\
\text { resultado. Duplicações } \\
\text { eliminadas }\end{array}$ & $\begin{array}{l}\text { Organiza os resultados em } \\
\text { uma ampla lista, } \\
\text { classificada por pontos. Esta } \\
\text { classificação é baseada nas } \\
\text { utiizadas pelos próprios } \\
\text { pesquisadores. }\end{array}$ & $\begin{array}{c}\text { Os resultados são } \\
\text { classificados em grupo de } \\
\text { palavras utilizados na } \\
\text { pesquisa. É possível } \\
\text { escolher a classificação } \\
\text { por domínio. }\end{array}$ \\
\hline $\begin{array}{l}\text { Permite lógica } \\
\text { booleana? }\end{array}$ & $\begin{array}{l}\text { Utiliza sempre AND. Permite } \\
\text { a utilização do OR, NOT e } \\
\text { ( ). Tenta encontrar termos } \\
\text { equivalentes para cada } \\
\text { ferramenta. Apresenta bons } \\
\text { resultados nos pesquisadores } \\
\text { Alta Vista, HotBot e Excite. } \\
\text { Nos demais seu desempenho é } \\
\text { sofrível. }\end{array}$ & $\begin{array}{l}\text { Utiliza sempre AND. É } \\
\text { possível utilizar OR, NOT } \\
\text { e ( ). Oferece bons } \\
\text { resultados nas ferramentas } \\
\text { de pesquisa que o apoiam } \\
\text { (Alta Vista, Excite). Nas } \\
\text { demais ferramentas pode } \\
\text { gerar lixo. }\end{array}$ & $\begin{array}{l}\text { Utiliza sempre AND. Pode } \\
\text { utilizar palavras como ANY } \\
\text { ou frases exatas. Utiliza os } \\
\text { termos booleanos, porém } \\
\text { resulta quase sempre em } \\
\text { lixo. }\end{array}$ & $\begin{array}{l}\text { Utiliza sempre AND. Para } \\
\text { os parâmetros OR, NOT, } \\
\text { ou ( ) tenta adaptá-los a } \\
\text { seus equivalentes em todas } \\
\text { as ferramentas. Os } \\
\text { resultados apresentam } \\
\text { sucesso variável. }\end{array}$ \\
\hline Utiliza +/-? & $\begin{array}{l}\text { Sim. Transforma em AND } \\
\text { para pesquisadores que não } \\
\text { aceitam o parâmetro }+ \text {. }\end{array}$ & $\begin{array}{l}\text { Sim. Maioria das } \\
\text { ferramentas que utiliza } \\
\text { aceitam. }\end{array}$ & $\begin{array}{l}\text { Utiliza mas não muito } \\
\text { eficientemente. }\end{array}$ & $\begin{array}{l}\text { Sim. Transforma em AND } \\
\text { para as ferramentas que não } \\
\text { aceitam o parâmetro }+ \text {. }\end{array}$ \\
\hline $\begin{array}{l}\text { Permite frases } \\
\text { entre aspas? }\end{array}$ & $\begin{array}{l}\text { Utiliza em alguns, mas não } \\
\text { em todos os pesquisadores } \\
\text { possíveis. }\end{array}$ & $\begin{array}{l}\text { O resultado parece incluir } \\
\text { frases e termos dentro da } \\
\text { frase }\end{array}$ & $\begin{array}{l}\text { Utiliza. Gera lixo onde não } \\
\text { é aceito. }\end{array}$ & $\begin{array}{l}\text { Utiliza. Gera lixo onde } \\
\text { não é aceito. }\end{array}$ \\
\hline
\end{tabular}

Extraído do site da Universidade da Califórnia, Berkeley

Fonte:http://www.lib.berkeley.edu/TeachingLib/Guides/Internet/MetaSearch.html

\section{OUTROS PESQUISADORES}

É possível, ainda, localizar informações na Internet utilizando-se outros pesquisadores mais específicos. São sites especializados que cobrem os mais diversos interesses tais como: coleção de links selecionados cobrindo tópicos específicos (webliographies), pesquisadores restritos a determinado assunto, dicionários on line, ferramentas que buscam informações em textos integrais de jornais e revistas, sites especializados na localização de endereços eletrônicos, empresas, etc. As possibilidades são infinitas. Abaixo seguem alguns exemplos. 
- Deja News(http://www.dejanews.com) Site especializado em localizar grupos de discussão. É possível buscar um grupo através da palavras chave ou simplesmente percorrendo a relação catalogada.

- Liszt(http://www.liszt.com)

Pesquisa listas de discussão por assunto e por palavra chave.

- Super Mail

(http://www.supermail.com.br)

Catálogo de endereços eletrônicos no Brasil.

- LookSmart

(http://www.looksmart.com)

Pesquisa em sites selecionados, classificados em categorias.

- Family Net Shepherd (http://family.netshepherd.com)

Mecanismo de busca que apresenta somente os sites considerados familiares. Um grupo de voluntários visitou milhares de sites construindo um banco de dados composto de sites considerados adequados. A busca é feita através do Alta Vista. Quando realiza uma pesquisa, compara os resultados apresentados com seu banco de dados disponibilizando, somente, os sites aprovados.

- Filez(http://www.filez.com)

Localiza exclusivamente sites que permitem transferência de arquivos (FTP).

- Research-it (http://www.iTools.com/research-it) Pesquisa nos principais dicionários e sites que realizam traduções. Localiza, ainda, bibliotecas, mapas, telefones, informações financeiras, códigos postais, etc.

- BizWeb (http://www.bizweb.com) Guia com mais de 30 mil empresas classificadas em 192 categorias.

- Europages (http://www.europages.com Dados de 150.000 empresas de 25 países europeus. Pode-se buscar por setor de atividade ou por nome da empresa.
Também tem informações econômicas e de negócios.

- EXPOguide Home Page (http://www.expoguide.com)

Feiras e conferências do mundo. Pode-se pesquisar pelo nome, data e país. Oferece informações detalhadas tais como: exibidores, taxas, espaço físico, etc.

- Human Search (http://www.humansearch.com) A pesquisa é feita por pessoas. Envia-se uma pergunta e um time de pesquisadores humanos tenta encontrar a resposta dentro ou fora da Internet. A resposta pode demorar 48 horas.

- Study Web (http://www.studyweb.com)

Editores do Study Web categorizaram e revisaram 20 mil sites acadêmicos ou de pesquisa.

É possível, através do Yahoo, localizar uma webliographies sobre um assunto específico digitando-se "assunto" AND indices na caixa de pesquisa (ou + "assunto" +indices). Encontrar mecanismo de busca dedicado a determinado tema pesquisando "assunto" AND ("web search" OR "internet search"). Procure, ainda, no Librarians' Index to the Internet (http://sunsite.berkeley.edu/ InternetIndex 2 , no Argus Clearinghouse (http:// www.clearinghouse.net), na Fisk University WWW Virtual Library (http://vlib.stanford.edu/ Overview.html), etc.

\section{CONSIDERAÇŌES FINAIS}

De modo geral pode-se adotar os seguintes princípios para a localização de informações na Internet:

- Escolha alguns pesquisadores e especialize-se nas suas formas de funcionamento para ser capaz de extrair todo seu potencial.

- Tente encontrar os pesquisadores que mais se adaptem as suas necessidades. Aprenda a analisar seu tópico para tirar vantagem das características dos diferentes mecanismos de busca. 
- Não limite a sua pesquisa a uma única ferramenta. Lembre-se que os resultados apresentados por diferentes pesquisadores podem ser bastante diversos. Metapesquisadores podem ser úteis já que buscam, simultaneamente, em várias ferramentas de busca.

- Defina, pelo menos, um catálogo e um índice já que indexam as informações utilizando diferentes critérios. Deste modo a pesquisa será mais abrangente e a probabilidade de encontrar o que se procura aumentará.

Endereços (URL) dos sites citados no texto

\section{Metapesquisadores}

- Dogpile: http://www.dogpile.com

- Inference Find: http://www.inference.com/ infind

- MetaCrawler: http://www.metacrawler.com

- Metafind:http://www.metafind.com

Catálogos e Índices

- Alta Vista:http://www.altavista.digital.com

- BookMarks:http://www.bookmarks.com.br

- Cadê: http://www.cade.com.br
- Excite: http://www.excite.com

- HotBot: http://www.hotbot.com

- Infoseek: http://www.infoseek.com

- Lycos: http://www.lycos.com

- Northern Light:http://www.northernlight.com

- Onde Ir?: http://www.ondeir.com.br

- Radar UOL: http://www.radaruol.com.br

- Surf:http://www.surf.com.br

- WebCrawler: http://www.webcrawler.com

- Yahoo!: http://www.yahoo.com

\section{BIBILIOGRAFIA}

ATHENIA Associates. Search Engines. http:// www.webreference.com/content/search/ bkground.html.

BAUER, M. Navegar sem naufragar.Revista Info Exame. Janeiro de 1998.

CONTE, R. Faróis digitais. Revista Internet World. Maio de 1998.

DANNY Sullivan, Editor.Search Engine Watch. http:// www.searchenginewatch.com Mecklermedia.

TEACHING Lybrary Internet Workshops, Find Information on the Internet: a tutorial. http:// www.lib.berkeley.edu/TeachingLib/Guides/ Internet/FindInfo.html University of California, Berkeley.

VENDITTO, G. Quem procura acha. Revista Internet World. Maio de 1998. 